\title{
An environmentally-friendly base organocatalyzed one-pot strategy for the regioselective synthesis of novel 3,6-diaryl-4-methylpyridazines
}

\author{
Mehdi Rimaz*, Farkhondeh Aali \\ Department of Chemistry, Payame Noor University, PO Box 19395-3697, Tehran, Iran
}

\section{A R T I C L E I N F O}

Article history:

Received 7 January 2016

Accepted 29 January 2016

Published 5 April 2016

\section{Keywords:}

1,4-Diazabicyclo[2.2.2] octane (DABCO)

Propiophenone

Arylglyoxalmonohydrate

Pyridazine

\begin{abstract}
A B S T R A C T
This report describes a new three-component strategy for the regioselective synthesis of a series of tri-substituted pyridazines via a 1,4-diazabicyclo[2.2.2] octane (DABCO)-catalyzed condensation of propiophenones, arylglyoxalmonohydrates and hydrazine hydrate in water. This method provides a green and convenient one-pot route toward a diverse set of 3,6-diaryl-4-methylpyridazines bearing various aryl substituents. This procedure is highly regioselective, operationally simple, uses water as a safe, environmentally friendly solvent, and DABCO as a green base-organocatalyst, and affords good to excellent yields of products.
\end{abstract}

(C) 2016, Dalian Institute of Chemical Physics, Chinese Academy of Sciences. Published by Elsevier B.V. All rights reserved.

\section{Introduction}

Nitrogen-containing heterocycle pyridazine is a key intermediate in the synthesis of several fused heterocycles used in drug discovery [1]. Recently, pyridazines have been considered by GlaxoSmithKline to be one of the "most developable" heteroaromatic rings for drug design [2]. Pyridazine analogues have proven to be useful ligands for different targets, and have been proposed as "privileged structures" for drug discovery [3]. Several compounds with pyridazine rings demonstrate biological activity (Fig. 1, 1-4), and there are many examples of naturally occurring pyridazines [4-7]. Pyridazines have also been recognized as selective GABA-A receptor antagonists, such as minaprine 1 [8]. Volonterio et al. [9] developed a synthesis of pyridazine-based scaffolds such as $\mathbf{2}$ to target protein/protein interaction as $\alpha$-helix mimetics, and 3-amino-6-aryl-pyridazines are also considered to be an interesting pharmacophore in drug discovery. Some pyridazines show biological activity in a range of disease areas including obesity [10], neu- rodegenerative diseases [11], and inflammatory pain, e.g. the selective CB2 agonist 3 [12]. Several pyridazine-containing compounds have also been identified as kinase inhibitors, and compound $\mathbf{4}$ has been identified as a potent p38 MAPK inhibitor [13].

Multicomponent reactions (MCRs) are capable of achieving high levels of diversity in a concise transformation, as they involve more than two building blocks to be combined in practical, time-saving, one-pot operations. These reactions are perfectly suited to automated synthesis, and have attracted considerable interest owing to their exceptional synthetic efficiency, inherent simple experimental procedures, and their one-pot nature [14-17]. Typically, the purification of products resulting from MCRs is also facile, as all the organic reagents involved are consumed and incorporated into the target compound $[18,19]$. MCRs leading to interesting heterocyclic scaffolds are particularly useful for the construction of diverse chemical libraries of drug-like molecules.

The Paal-Knorr synthesis is one of the most common ap-

* Corresponding author. Tel: +98-443-5625711; Fax: +98-443-5666999; E-mail: rimaz.mehdi@gmail.com DOI: 10.1016/S1872-2067(15)61060-9 | http://www.sciencedirect.com/science/journal/18722067 | Chin. J. Catal., Vol. 37, No. 4, April 2016 
<smiles>Cc1cc(-c2ccccc2)nnc1NCCN1CCOCC1</smiles><smiles>CC(C)Cc1cc(-c2ccc(Oc3ccccc3)cc2)nnc1-c1nnc(C(C)C)o1</smiles>

Alpha-helix mimetics<smiles>[CH]C1CCC(C)N1c1ccc(-c2cncc3ccccc23)nn1</smiles>

3

Selective $\mathrm{CB}_{2}$ agonist

Fig. 1. Selected biologically active substituted pyridazines.

proaches for the construction various five- or six-membered heterocycles. In the Paal-Knorr synthesis of pyridazines, 1,4-dicarbonyl compounds are converted to pyridazines via a dehydrative cyclization in the presence of hydrazine, and subsequent oxidation [20-25].

As part of our ongoing program to develop efficient and robust MC methods for the preparation of heterocyclic compounds [25-30], we sought to develop a convenient preparation of 3,6-diaryl substituted 4-methylpyridazines 5-38 via a regioselective one-pot condensation reaction of substituted propiophenones 39a-d with arylglyoxalmonohydrates $\mathbf{4 0 a - j}$ and hydrazine in the presence of catalytic amounts of 1,4-diazabicyclo[2.2.2] octane (DABCO), 1,8-diazabicyclo[5.4.0] undec-7-ene (DBU) or 1,5-diazabicyclo[4.3.0]non-7-ene (DBN) as shown in Scheme 1. To the best of our knowledge, there are no reports in the literature for the formation of pyridazine derivatives via base-organocatalyzed condensation of propiophenones with arylglyoxalmonohydrates in the presence of hydrazine hydrate.

\section{Experimental}

2.1. General procedures for the regioselective DABCO-catalyzed one-pot synthesis of 3,6-diaryl-4-methylpyridazine derivatives

To a mixture of arylglyoxalmonohydrate (1 mmol), propiophenone (1 mmol) and DABCO (50 mol\%) in water $(10 \mathrm{~mL})$ were added hydrazine hydrate $(4 \mathrm{mmol})$. The suspension was stirred at $25{ }^{\circ} \mathrm{C}$ until precipitation ceased (2-4 h). After completion of the reaction, the mixture was filtered and purified by recrystallization from ethanol.

\subsection{Analytical data for the products}

3-(3-Bromophenyl)-6-phenyl-4-methylpyridazine (5): white crystals; 86\%; mp $115{ }^{\circ} \mathrm{C}$. IR (KBr): $v_{\max }=3063,2971,2929$, $1577,1392,1261,1042,886 \mathrm{~cm}^{-1}$. ${ }^{1} \mathrm{H}$ NMR (300 MHz, $\left.\mathrm{CDCl}_{3}\right) \delta$ $2.45(\mathrm{~s}, 3 \mathrm{H}), 7.39(\mathrm{t}, 1 \mathrm{H}, J=7.8), 7.47-7.67(\mathrm{~m}, 5 \mathrm{H}), 7.74(\mathrm{~s}, 1 \mathrm{H})$, $7.84(\mathrm{~s}, 1 \mathrm{H}), 8.14(\mathrm{~d}, 2 \mathrm{H}, J=6.3) .{ }^{13} \mathrm{C}$ NMR $\left(75 \mathrm{MHz}^{\mathrm{CDCl}} \mathrm{CDC}_{3} \delta\right.$ $19.9,122.5,124.4,124.7,126.4,127.8,128.8,129.8,131.0$, 132.8, 133.3, 136.1, 139.0, 157.8, 159.1. Anal. found, C, 62.83; H, 4.06; $\mathrm{N}, 8.68 . \mathrm{C}_{17} \mathrm{H}_{13} \mathrm{BrN}_{2}$ requires $\mathrm{C}, 62.79 ; \mathrm{H}, 4.03 ; \mathrm{N}, 8.61$.

3-(3-Bromophenyl)-6-(4-bromophenyl)-4-methylpyridazine (6): yellow crystals; $72 \%$; mp $157{ }^{\circ} \mathrm{C}$. IR (KBr): $v_{\max }=3083$, 3053, 2969, 1589, 1421, 1074, 1004, $850 \mathrm{~cm}^{-1}$. ${ }^{1} \mathrm{H}$ NMR $(300$ $\left.\mathrm{MHz}, \mathrm{CDCl}_{3}\right) \delta 2.46(\mathrm{~s}, 3 \mathrm{H}), 7.39(\mathrm{t}, 1 \mathrm{H}, J=8.1), 7.53-7.7(\mathrm{~m}, 4 \mathrm{H})$, 7.72 (s, 1H), $7.82(\mathrm{~s}, 1 \mathrm{H}), 8.02(\mathrm{~d}, 2 \mathrm{H}, J=8.1) .{ }^{13} \mathrm{C}$ NMR $(75 \mathrm{MHz}$, $\left.\mathrm{CDCl}_{3}\right) \delta 19.8,122.5,124.3,124.7,126.1,127.5,128.8,129.5$, 131.3, 133.1, 134.9, 136.3, 138.8, 157.7, 159.4. Anal. found, C, 50.57; $\mathrm{H}, 2.97 ; \mathrm{N}, 7.00 . \mathrm{C}_{17} \mathrm{H}_{12} \mathrm{Br}_{2} \mathrm{~N}_{2}$ requires $\mathrm{C}, 50.53$; $\mathrm{H}, 2.99$; $\mathrm{N}, 6.93$.

3-(3-Bromophenyl)-6-(4-chlorophenyl)-4-methylpyridazine (7): yellow crystals; 88\%; mp $176^{\circ} \mathrm{C}$. IR (KBr): $v_{\max }=3091$, 3057, 3032, 1586, 1414, 1386, 1089, $893 \mathrm{~cm}^{-1}$. ${ }^{1} \mathrm{H}$ NMR $(300$ $\left.\mathrm{MHz} \mathrm{CDCl}_{3}\right) \delta 2.46(\mathrm{~s}, 3 \mathrm{H}), 7.39(\mathrm{t}, 1 \mathrm{H}, J=7.8), 7.47-7.67(\mathrm{~m}$, 4H), $7.72(\mathrm{~s}, 1 \mathrm{H}), 7.82(\mathrm{~s}, 1 \mathrm{H}), 8.08(\mathrm{~d}, 2 \mathrm{H}, J=7.8) .{ }^{13} \mathrm{C}$ NMR $(75$ $\left.\mathrm{MHz}, \mathrm{CDCl}_{3}\right) \delta 19.8,122.6,124.4,126.1,127.3,128.4,128.8$, 129.2, 130.0, 131.1, 133.3, 136.2, 138.9, 156.7, 159.4. Anal. found, $\mathrm{C}, 56.79 ; \mathrm{H}, 3.32 ; \mathrm{N}, 7.85 . \mathrm{C}_{17} \mathrm{H}_{12} \mathrm{BrClN}_{2}$ requires $\mathrm{C}, 56.77$; H, 3.36; N, 7.79.

3-(3-Bromophenyl)-6-(4-fluorophenyl)-4-methylpyridazine (8): white crystals; 82\%; mp $141{ }^{\circ} \mathrm{C}$. IR (KBr): $v_{\max }=3122$, 3080, 3042, 2925, 1590, 1416, 1223, 1099, $843 \mathrm{~cm}^{-1}$. ${ }^{1} \mathrm{H}$ NMR (300 MHz, $\left.\mathrm{CDCl}_{3}\right) \delta 2.44(\mathrm{~s}, 3 \mathrm{H}), 7.17-7.3(\mathrm{~m}, 2 \mathrm{H}), 7.40(\mathrm{t}, 1 \mathrm{H}, J$ = 7.8), 7.53-7.67 (m, 2H), $7.72(\mathrm{~s}, 1 \mathrm{H}), 7.83(\mathrm{~s}, 1 \mathrm{H}), 8.08-8.19$ $(\mathrm{m}, 2 \mathrm{H}) .{ }^{13} \mathrm{C}$ NMR $\left(75 \mathrm{MHz}, \mathrm{CDCl}_{3}\right) \delta 19.9,114.9,115.2,116.9$, 117.2, 124.3, 126.1, 128.1, 128.8, 129.9, 131.2, 132.9, 136.2, 138.9, 156.8, 162.5. Anal. found, C, 54.54; H, 3.55; N, 8.20. $\mathrm{C}_{17} \mathrm{H}_{12} \mathrm{BrFN}_{2}$ requires $\mathrm{C}, 59.49 ; \mathrm{H}, 3.52 ; \mathrm{N}, 8.16$.

3-(3-Bromophenyl)-6-(4-methoxyphenyl)-4-methylpyridazine (9): white crystals; $78 \%$; mp $119{ }^{\circ} \mathrm{C}$. IR (KBr): $v_{\max }=3057$, 3015, 2960, 2939, 2842, 1589, 1428, 1249, 1034, $842 \mathrm{~cm}^{-1} .{ }^{1} \mathrm{H}$ NMR (300 MHz, CDCl $) \delta 2.47(\mathrm{~s}, 3 \mathrm{H}), 3.93(\mathrm{~s}, 3 \mathrm{H}), 7.06(\mathrm{~d}, 1 \mathrm{H}, J$ $=8.1), 7.34-7.5(\mathrm{~m}, 2 \mathrm{H}), 7.53-7.69(\mathrm{~m}, 3 \mathrm{H}), 7.54(\mathrm{~s}, 1 \mathrm{H})$,<smiles>CCC(=O)c1ccccc1</smiles>

39a-d

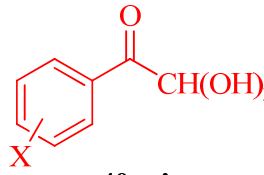

$40 \mathbf{a}-\mathbf{j}$

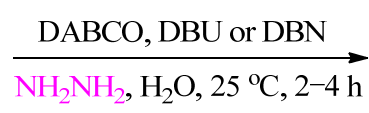

${ }_{2}$

$\mathrm{X}=4-\mathrm{H}, 4-\mathrm{Br}, 4-\mathrm{Cl}, 4-\mathrm{F}, 4-\mathrm{OCH}_{3}, 3-\mathrm{Br}, 3-\mathrm{OCH}_{3}, 3,4-\left(\mathrm{OCH}_{3}\right)_{2}, 3,4-\left(\mathrm{OCH}_{2} \mathrm{O}\right), 4-\mathrm{OH}-3-\mathrm{OCH}_{3}$ $\mathrm{Y}=4-\mathrm{Br}, 3-\mathrm{Br}, 4-\mathrm{Cl}, 4-\mathrm{CH}_{3}$

Scheme 1. Regioselective base-organocatalyzed one-pot synthesis of 3,6-diaryl-4-methylpyridazines. 
7.79-7.89 (m, 2H). ${ }^{13} \mathrm{C}$ NMR (75 MHz, $\left.\mathrm{CDCl}_{3}\right) \delta 19.9,56.2,110.9$, $113.0,115.3,118.4,120.2,124.8,128.8,131.0,132.9,136.1$, 137.4, 139.0, 159.3. Anal. found, C, 60.87; H, 4.23; N, 7.99. $\mathrm{C}_{18} \mathrm{H}_{15} \mathrm{BrN}_{2} \mathrm{O}$ requires $\mathrm{C}, 60.86 ; \mathrm{H}, 4.26 ; \mathrm{N}, 7.89$.

3,6-Bis(3-bromophenyl)-4-methylpyridazine (10): yellow crystals; $71 \%$; mp $109{ }^{\circ} \mathrm{C}$. IR (KBr): $\nu_{\max }=2960,2931,2859$, 1584, 1566, 1379, 1426, 1065, $883 \mathrm{~cm}^{-1} .{ }^{1} \mathrm{H}$ NMR (300 MHz, $\left.\mathrm{CDCl}_{3}\right) \delta 2.48(\mathrm{~s}, 3 \mathrm{H}), 7.36-7.48(\mathrm{~m}, 2 \mathrm{H}), 7.53-7.7(\mathrm{~m}, 3 \mathrm{H}), 7.74$ $(\mathrm{s}, 1 \mathrm{H}), 7.84(\mathrm{~s}, 1 \mathrm{H}), 8.09(\mathrm{~d}, 1 \mathrm{H}, J=7.8), 8.30(\mathrm{~s}, 1 \mathrm{H}) .{ }^{13} \mathrm{C} \mathrm{NMR}$ $\left(75 \mathrm{MHz}, \mathrm{CDCl}_{3}\right) \delta 20.0,123.7,126.5,129.4,129.7,130.2,130.9$, 131.4, 132.0, 133.0, 133.7, 136.5, 137.9, 156.2, 159.9. Anal. found, $\mathrm{C}, 50.54 ; \mathrm{H}, 2.31 ; \mathrm{N}, 7.06 . \mathrm{C}_{17} \mathrm{H}_{12} \mathrm{Br}_{2} \mathrm{~N}_{2}$ requires $\mathrm{C}, 50.53$; H, 2.99; N, 6.93 .

3-(3-Bromophenyl)-6-(3-methoxyphenyl)-4-methylpyridazine (11): white crystals; 70\%; mp $104{ }^{\circ} \mathrm{C}$. IR (KBr): $v_{\max }=3067$, 3008, 2971, 2920, 2849, 1589, 1467, 1377, 1038, 855, 773 $\mathrm{cm}^{-1} .{ }^{1} \mathrm{H}$ NMR $\left(300 \mathrm{MHz}, \mathrm{CDCl}_{3}\right) \delta 2.44(\mathrm{~s}, 3 \mathrm{H}), 3.89(\mathrm{~s}, 3 \mathrm{H}), 7.06$ (d, 2H, $J=8.7$ ), $7.38(\mathrm{t}, 1 \mathrm{H}, J=8.1), 7.54-7.65(\mathrm{~m}, 2 \mathrm{H}), 7.69$ (s, $1 \mathrm{H}), 7.83(\mathrm{~s}, 1 \mathrm{H}), 8.11$ (d, $2 \mathrm{H}, J=8.7) .{ }^{13} \mathrm{C} \mathrm{NMR}\left(75 \mathrm{MHz}, \mathrm{CDCl}_{3}\right.$ ) $\delta 20.9,56.1,113.3,113.3,115.4,115.5,123.8,125.6,127.5$, $128.8,130.8,135.9,139.2,157.3,158.6,161.3$. Anal. found, C, 60.83; $\mathrm{H}, 4.25 ; \mathrm{Br}, \mathrm{N}, 7.99 . \mathrm{C}_{18} \mathrm{H}_{15} \mathrm{BrN}_{2} \mathrm{O}$ requires $\mathrm{C}, 60.86 ; \mathrm{H}$, 4.26; N, 7.89.

3-(3-Bromophenyl)-6-(3,4-dimethoxyphenyl)-4-methylpyridazine (12): yellow crystals; $84 \%$; $\operatorname{mp~} 174{ }^{\circ} \mathrm{C}$. IR (KBr): $v_{\max }=$ 3066, 2998, 2939, 2842, 1587, 1422, 1021, 873, $768 \mathrm{~cm}^{-1} .{ }^{1} \mathrm{H}$ NMR (300 MHz, $\left.\mathrm{CDCl}_{3}\right) \delta 2.44(\mathrm{~s}, 3 \mathrm{H}), 3.96(\mathrm{~s}, 3 \mathrm{H}), 4.01(\mathrm{~s}, 3 \mathrm{H})$, $6.98(\mathrm{~d}, 1 \mathrm{H}, J=7.5), 7.38(\mathrm{t}, 1 \mathrm{H}, J=7.8), 7.70(\mathrm{~s}, 1 \mathrm{H}), 7.83(\mathrm{~s}$, 1H), $7.94(\mathrm{~s}, 1 \mathrm{H}) .{ }^{13} \mathrm{C}$ NMR $\left(75 \mathrm{MHz}, \mathrm{CDCl}_{3}\right) \delta 19.9,55.1,56.8$, $109.0,110.0,110.3,110.9,111.9,112.2,120.4,123.9,128.8$, 135.9, 139.1, 149.6, 150.8, 157.1, 158.7. Anal. found, C, 59.23; $\mathrm{H}$, 4.45; N, 7.27. $\mathrm{C}_{19} \mathrm{H}_{17} \mathrm{BrN}_{2} \mathrm{O}_{2}$ requires $\mathrm{C}, 59.27 ; \mathrm{H}, 4.46 ; \mathrm{N}, 7.39$.

3-(3-Bromophenyl)-6-(3,4-methylenedioxyphenyl)-4-methylpyridazine (13): yellow crystals, $80 \%$; $\mathrm{mp} 153{ }^{\circ} \mathrm{C}$. IR (KBr): $\nu_{\max }$ = 3071, 2996, 2912, 2786, 1586, 1491, 1256, 1038, $874 \mathrm{~cm}^{-1}$. ${ }^{1} \mathrm{H}$ NMR $\left(300 \mathrm{MHz}, \mathrm{CDCl}_{3}\right) \delta 2.43(\mathrm{~s}, 3 \mathrm{H}), 6.06(\mathrm{~s}, 2 \mathrm{H}), 6.96(\mathrm{~d}, J$ $=8.1,1 \mathrm{H}), 7.38(\mathrm{t}, J=8.1,1 \mathrm{H}), 7.68-7.53(\mathrm{~m}, 4 \mathrm{H}), 7.72(\mathrm{~s}, 1 \mathrm{H})$, 7.82 (s, 1H). ${ }^{13} \mathrm{C}$ NMR (75 MHz, $\left.\mathrm{CDCl}_{3}\right) \delta 19.8,101.5,102.9$, $106.4,107.5,107.8,108.2,109.5,122.2,124.1,125.9,128.8$, 136.0, 139.1, 148.6, 149.3, 157.2, 158.8. Anal. found, C, 58.59; $\mathrm{H}$, 3.49; N, 7.77. $\mathrm{C}_{18} \mathrm{H}_{13} \mathrm{BrN}_{2} \mathrm{O}_{2}$ requires $\mathrm{C}, 58.56 ; \mathrm{H}, 3.55 ; \mathrm{N}, 7.59$.

3-(3-Bromophenyl)-6-(4-hydroxy-3-methoxyphenyl)-4methylpyridazine (14): white crystals; $74 \%$; mp $179{ }^{\circ} \mathrm{C}$. IR $(\mathrm{KBr}): v_{\max }=3328,3083,3057,2969,1589,1421,1398,1389$, $1074,1005,829,697 \mathrm{~cm}^{-1}$. ${ }^{1} \mathrm{H}$ NMR $\left(300 \mathrm{MHz}, \mathrm{CDCl}_{3}\right) \delta 2.45(\mathrm{~s}$, $3 \mathrm{H}), 4.03(\mathrm{~s}, 3 \mathrm{H}), 5.93(\mathrm{~s}, 1 \mathrm{H}), 7.39(\mathrm{~d}, J=8.4,1 \mathrm{H}), 7.49(\mathrm{t}, J=$ 8.4, 1H), 7.63-7.58 (m, 2H), $7.71(\mathrm{~s}, 1 \mathrm{H}), 7.85(\mathrm{~s}, 1 \mathrm{H}), 8.00(\mathrm{~s}$, 1H). ${ }^{13} \mathrm{C}$ NMR (75 MHz, $\left.\mathrm{CDCl}_{3}\right) \delta 19.9,57.0,108.5,110.5,113.5$, $119.0,121.2,123.8,128.2,130.9,132.8,135.8,139.1,146.3$, 147.8, 158.9, 159.3. Anal. found, C, 58.23; H, 4.05; N, 7.76. $\mathrm{C}_{18} \mathrm{H}_{15} \mathrm{BrN}_{2} \mathrm{O}_{2}$ requires $\mathrm{C}, 58.24 ; \mathrm{H}, 4.07 ; \mathrm{Br}, \mathrm{N}, 7.55$.

3-(4-Bromophenyl)-6-phenyl-4-methylpyridazine (15): white crystals; $78 \%$; mp $163{ }^{\circ} \mathrm{C}$. IR (KBr): $v_{\max }=3055,2930$, 1587, 1445, 1389, 1075, 1004, $830 \mathrm{~cm}^{-1} .{ }^{1} \mathrm{H}$ NMR $(300 \mathrm{MHz}$, $\left.\mathrm{CDCl}_{3}\right): \delta 2.44(\mathrm{~s}, 3 \mathrm{H}), 7.46-7.58(\mathrm{~m}, 5 \mathrm{H}), 7.65(\mathrm{~d}, 2 \mathrm{H}, J=8.1)$, $7.74(\mathrm{~s}, 1 \mathrm{H}), 8.13(\mathrm{~d}, 2 \mathrm{H}, J=6.3) .{ }^{13} \mathrm{C} \mathrm{NMR}\left(75 \mathrm{MHz}, \mathrm{CDCl}_{3}\right) \delta$ $19.9,126.2,126.5,127.4,127.9,129.6,130.8,131.6,132.3$,
135.7, 136.2, 157.6, 159.5. Anal. found, C, 62.81; H, 4.05; N, 8.72. $\mathrm{C}_{17} \mathrm{H}_{13} \mathrm{BrN}_{2}$ requires $\mathrm{C}, 62.79 ; \mathrm{H}, 4.03 ; \mathrm{N}, 8.61$.

3,6-Bis(4-bromophenyl)-4-methylpyridazine (16): yellow crystals; $72 \%$; $\mathrm{mp} 174{ }^{\circ} \mathrm{C}$. IR (KBr): $v_{\max }=3085,1590,1483$, 1401, 1072, 1002, $822 \mathrm{~cm}^{-1} .{ }^{1} \mathrm{H}$ NMR (300 MHz, $\left.\mathrm{CDCl}_{3}\right) \delta 2.47$ (s, 3H), $7.55(\mathrm{~d}, J=8.4,2 \mathrm{H}), 7.61-7.73(\mathrm{~m}, 4 \mathrm{H}), 7.74(\mathrm{~s}, 1 \mathrm{H}), 8.02$ $(\mathrm{d}, 2 \mathrm{H}, J=8.4) .{ }^{13} \mathrm{C} \mathrm{NMR}\left(75 \mathrm{MHz}, \mathrm{CDCl}_{3}\right) \delta 20.0,124.9,127.7$, 129.5, 129.7, 130.2, 130.9, 131.6, 132.0, 132.4, 133.0, 156.6, 159.6. Anal. found, C, 50.55; H, 2.96; N, 7.04. $\mathrm{C}_{17} \mathrm{H}_{12} \mathrm{Br}_{2} \mathrm{~N}_{2}$ requires $\mathrm{C}, 50.53 ; \mathrm{H}, 2.99 ; \mathrm{N}, 6.93$.

3-(4-Bromophenyl)-6-(4-chlorophenyl)-4-methylpyridazine (17): white crystals; $88 \%$; mp $176{ }^{\circ} \mathrm{C}$. IR (KBr): $v_{\max }=3095$, 3030, 1593, 1483, 1400, 1073, 1006, $824 \mathrm{~cm}^{-1}$. ${ }^{1} \mathrm{H}$ NMR $(300$ $\left.\mathrm{MHz} \mathrm{CDCl}_{3}\right) \delta 2.44(\mathrm{~s}, 3 \mathrm{H}), 7.46-7.58(\mathrm{~m}, 4 \mathrm{H}), 7.66(\mathrm{~d}, 2 \mathrm{H}, J=$ 8.4), $7.71(\mathrm{~s}, 1 \mathrm{H}), 8.08(\mathrm{~d}, 2 \mathrm{H}, J=8.4) .{ }^{13} \mathrm{C} \mathrm{NMR}\left(75 \mathrm{MHz}, \mathrm{CDCl}_{3}\right)$ $\delta$ 19.9, 124.6, 126.1, 128.1, 129.1, 129.8, 130.8, 131.6, 132.4, 135.6, 136.2, 156.5, 159.6. Anal. found, C, 56.80; H, 3.38; N, 7.89. $\mathrm{C}_{17} \mathrm{H}_{12} \mathrm{BrClN}_{2}$ requires $\mathrm{C}, 56.77 ; \mathrm{H}, 3.36 ; \mathrm{N}, 7.79$.

3-(4-Bromophenyl)-6-(4-fluorophenyl)-4-methylpyridazine (18): white crystals; $92 \%$; $\operatorname{mp} 188^{\circ} \mathrm{C}$. IR (KBr): $v_{\max }=3080$, $2975,1593,1505,1379,1228,1071,1001,831 \mathrm{~cm}^{-1} .{ }^{1} \mathrm{H}$ NMR $\left(300 \mathrm{MHz}, \mathrm{CDCl}_{3}\right) \delta 2.44(\mathrm{~s}, 3 \mathrm{H}), 7.16-7.27(\mathrm{~m}, 2 \mathrm{H}), 7.53(\mathrm{~d}, 2 \mathrm{H}$, $J=8.4), 7.64(\mathrm{~d}, 2 \mathrm{H}, J=8.1), 7.71(\mathrm{~s}, 1 \mathrm{H}), 8.08-8.18(\mathrm{~m}, 2 \mathrm{H}) \cdot{ }^{13} \mathrm{C}$ NMR (75 MHz, $\left.\mathrm{CDCl}_{3}\right) \delta 19.9,114.9,115.2,116.8,117.1,128.2$, $129.9,130.8,132.4,156.6,159.4,162.4,165.7$. Anal. found, C, 54.51; $\mathrm{H}, 3.53$; N, 8.28. $\mathrm{C}_{17} \mathrm{H}_{12} \mathrm{BrFN}_{2}$ requires $\mathrm{C}, 59.49 ; \mathrm{H}, 3.52$; $\mathrm{N}, 8.16$.

3-(4-Bromophenyl)-6-(4-methoxyphenyl)-4-methylpyridazine (19): white crystals; $90 \%$; $\mathrm{mp} 133{ }^{\circ} \mathrm{C}$. IR (KBr): $v_{\max }=3000$, 2970, 2930, 2835, 1589, 1492, 1399, 1256, 1034, 1000, 835 $\mathrm{cm}^{-1} .{ }^{1} \mathrm{H}$ NMR (300 MHz, CDCl $) \delta 2.42(\mathrm{~s}, 3 \mathrm{H}), 3.88(\mathrm{~s}, 3 \mathrm{H}), 7.04$ (d, 2H, $J=8.1$ ), 7.53 (d, 2H, $J=7.8$ ), $7.62(\mathrm{~d}, 2 \mathrm{H}, J=7.8$ ), 7.67 (s, $1 \mathrm{H}), 8.09(\mathrm{~d}, 2 \mathrm{H}, J=8.1) .{ }^{13} \mathrm{C}$ NMR $\left(75 \mathrm{MHz} \mathrm{CDCl}_{3}\right) \delta 19.9,56.0$, 113.3, 113.8, 114.7, 115.5, 127.6, 129.3, 130.7, 132.0, 132.3, 157.0, 158.9, 161.3. Anal. found, C, 60.89; H, 4.22; N, 8.03. $\mathrm{C}_{18} \mathrm{H}_{15} \mathrm{BrN}_{2} \mathrm{O}$ requires $\mathrm{C}, 60.86 ; \mathrm{H}, 4.26 ; \mathrm{N}, 7.89$.

3-(4-Bromophenyl)-6-(3-bromophenyl)-4-methylpyridazine (20): white crystals; 71\%; mp $132{ }^{\circ} \mathrm{C}$. IR (KBr): $v_{\max }=3055$, 3010, 1590, 1566, 1375, 1047, 1000, $886 \mathrm{~cm}^{-1}$. ${ }^{1} \mathrm{H}$ NMR $(300$ $\left.\mathrm{MHz} \mathrm{CDCl}_{3}\right) \delta 2.46(\mathrm{~s}, 3 \mathrm{H}), 7.40(\mathrm{t}, 1 \mathrm{H}, J=8.1), 7.54(\mathrm{~d}, 2 \mathrm{H}, J=$ 8.4), 7.59-7.71 (m, 3H), $7.72(\mathrm{~s}, 1 \mathrm{H}), 8.07$ (d, 1H, $J=7.8), 8.29$ (s, 1H). $\left.{ }^{13} \mathrm{C} \mathrm{NMR} \mathrm{(75} \mathrm{MHz,} \mathrm{CDCl}_{3}\right) \delta 19.9,123.7,126.5,129.4$, 129.7, 130.2, 130.9, 131.4, 132.0, 133.0, 133.7, 136.5, 137.9, 156.2, 159.9. Anal. found, C, 50.57; H, 2.30; N, 7.10. $\mathrm{C}_{17} \mathrm{H}_{12} \mathrm{Br}_{2} \mathrm{~N}_{2}$ requires $\mathrm{C}, 50.53 ; \mathrm{H}, 2.99 ; \mathrm{N}, 6.93$.

3-(4-Bromophenyl)-6-(3,4-dimethoxyphenyl)-4-methylpyridazine (21): yellow crystals; $91 \%$; mp $174{ }^{\circ} \mathrm{C}$. IR (KBr): $v_{\max }=$ 2935, 2830, 1604, 1587, 1401, 1091, 1023, $838 \mathrm{~cm}^{-1} .{ }^{1} \mathrm{H}$ NMR

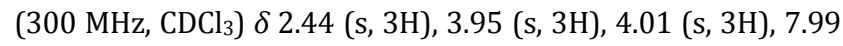
(d, $1 \mathrm{H}, J=8.4$ ), 7.45-7.6 (m, 3H), $7.64(\mathrm{~d}, 2 \mathrm{H}, J=8.4), 7.71(\mathrm{~s}$, 1H), $7.94(\mathrm{~s}, 1 \mathrm{H}) .{ }^{13} \mathrm{C} \mathrm{NMR}\left(75 \mathrm{MHz}, \mathrm{CDCl}_{3}\right) \delta 20.0,56.7,57.3$, 109.0, 109.9, 110.4, 110.8, 111.8, 112.2, 130.1, 131.6, 132.0, 136.3, 149.5, 150.9, 156.9, 159.0. Anal. found, C, 59.30; H, 4.48; $\mathrm{N}, 7.42 . \mathrm{C}_{19} \mathrm{H}_{17} \mathrm{BrN}_{2} \mathrm{O}_{2}$ requires $\mathrm{C}, 59.27 ; \mathrm{H}, 4.46 ; \mathrm{N}, 7.39$.

3-(4-Bromophenyl)-6-(3,4-methylenedioxyphenyl)-4-methylpyridazine (22): yellow crystals; $79 \%$; $\operatorname{mp} 166^{\circ} \mathrm{C}$. IR (KBr): $v_{\max }$ $=3075,2900,1592,1504,1451,1250,1107,1001,818 \mathrm{~cm}^{-1}$. 
${ }^{1} \mathrm{H}$ NMR (300 MHz, CDCl $) \delta 2.42(\mathrm{~s}, 3 \mathrm{H}), 6.05(\mathrm{~s}, 2 \mathrm{H}), 6.94(\mathrm{~d}$, $1 \mathrm{H}, J=8.1$ ), $7.52(\mathrm{~d}, 2 \mathrm{H}, J=8.1), 7.56-7.69(\mathrm{~m}, 4 \mathrm{H}), 7.70(\mathrm{~s}, 1 \mathrm{H})$. ${ }^{13} \mathrm{C}$ NMR $\left(75 \mathrm{MHz}, \mathrm{CDCl}_{3}\right) \delta 19.9,101.5,106.5,107.9,108.1$, 109.4, 122.1, 126.0, 129.7, 130.8, 131.6, 132.3, 136.2, 148.5, 157.0, 159.1. Anal. found, C, 58.56; $\mathrm{H}, 3.53 ; \mathrm{N}, 7.69 . \mathrm{C}_{18} \mathrm{H}_{13} \mathrm{BrN}_{2} \mathrm{O}_{2}$ requires $\mathrm{C}$, 58.56; $\mathrm{H}, 3.55 ; \mathrm{N}, 7.59$.

3-(4-Chlorophenyl)-6-phenyl-4-methylpyridazine (23): white crystals; $28 \%$; $m p 163{ }^{\circ} \mathrm{C}$. IR (KBr): $v_{\max }=3087,3070$, 3041, 2927, 1597, 1587, 1490, 1451, 1092, 1006, $789 \mathrm{~cm}^{-1} .{ }^{1} \mathrm{H}$ NMR (300 MHz, $\left.\mathrm{CDCl}_{3}\right) \delta 2.44(\mathrm{~s}, 3 \mathrm{H}), 7.53-7.47(\mathrm{~m}, 5 \mathrm{H}), 7.62$ (d, $2 \mathrm{H}, J=8.4), 7.73(\mathrm{~s}, 1 \mathrm{H}), 8.13(\mathrm{~d}, 2 \mathrm{H}, J=6.3) .{ }^{13} \mathrm{C}$ NMR $(75$ $\left.\mathrm{MHz}_{\mathrm{CDCl}}\right) \delta 19.9,124.6,126.0,127.5,128.8,129.7,129.8$, 130.1, 131.6, 135.0, 136.0, 157.6, 159.4. Anal. found, C, 72.77; H, 4.69; $\mathrm{N}, 10.17$. $\mathrm{C}_{17} \mathrm{H}_{13} \mathrm{ClN}_{2}$ requires $\mathrm{C}, 72.73 ; \mathrm{H}, 4.67 ; \mathrm{N}, 9.98$.

3-(4-Chlorophenyl)-6-(4-bromophenyl)-4-methylpyridazine (24): white crystals; $67 \%$; $\mathrm{mp} 174{ }^{\circ} \mathrm{C}$. IR (KBr): $v_{\max }=3090$, 3055, 3035, 2960, 2925, 1592, 1486, 1404, 1091, 1004, 824 $\mathrm{cm}^{-1} .{ }^{1} \mathrm{H}$ NMR (300 MHz, $\left.\mathrm{CDCl}_{3}\right) \delta 2.45(\mathrm{~s}, 3 \mathrm{H}), 7.50(\mathrm{~d}, 2 \mathrm{H}, J=$ 8.4), 7.57-7.69 (m, 4H), $7.72(\mathrm{~s}, 1 \mathrm{H}), 8.02$ (d, $2 \mathrm{H}, J=8.4) .{ }^{13} \mathrm{C}$ NMR $\left(75 \mathrm{MHz}, \mathrm{CDCl}_{3}\right) \delta 20.0,124.6,126.0,127.6,128.1,129.8$, $129.3,131.5,132.9,135.2,136.2,156.5,159.7$. Anal. found, C, 56.81; H, 3.35; N, 7.95. $\mathrm{C}_{17} \mathrm{H}_{12} \mathrm{BrClN}_{2}$ requires $\mathrm{C}, 56.77$; $\mathrm{H}, 3.36$; N, 7.79 .

3,6-Bis(4-chlorophenyl)-4-methylpyridazine (25): white crystals; $66 \%$; $\mathrm{mp} 181^{\circ} \mathrm{C}$. IR (KBr): $v_{\max }=3090,3060,3030$, 1587, 1487, 1376, 1090, 1008, $827 \mathrm{~cm}^{-1}$. ${ }^{1} \mathrm{H}$ NMR (300 MHz, $\left.\mathrm{CDCl}_{3}\right) \delta 2.46(\mathrm{~s}, 3 \mathrm{H}), 7.47-7.56(\mathrm{~m}, 4 \mathrm{H}), 7.62(\mathrm{~d}, 2 \mathrm{H}, J=8.1)$, 7.72 (s, 1H), 8.09 (d, 2H, $J=8.4$ ). ${ }^{13} \mathrm{C}$ NMR (75 MHz, $\left.\mathrm{CDCl}_{3}\right) \delta$ 20.0, 124.6, 126.0, 127.4, 127.5, 128.1, 129.1, 129.8, 130.4, $131.3,136.2,156.5,159.6$. Anal. found, C, 64.80; H, 3.81; N, 9.03. $\mathrm{C}_{17} \mathrm{H}_{12} \mathrm{Cl}_{2} \mathrm{~N}_{2}$ requires $\mathrm{C}$, 64.78; $\mathrm{H}, 3.84 ; \mathrm{N}, 8.89$.

3-(4-Chlorophenyl)-6-(4-fluorophenyl)-4-methylpyridazine (26): white crystals; $81 \%$; $m p 182{ }^{\circ} \mathrm{C}$. IR (KBr): $v_{\max }=3090$, 3080, 2970, 2925, 2855, 1596, 1487, 1389, 1089, 1003, 842 $\mathrm{cm}^{-1} .{ }^{1} \mathrm{H} \mathrm{NMR}\left(300 \mathrm{MHz}, \mathrm{CDCl}_{3}\right) \delta 2.45(\mathrm{~s}, 3 \mathrm{H}), 7.16-7.29(\mathrm{~m}$, $2 \mathrm{H}), 7.50(\mathrm{~d}, 2 \mathrm{H}, J=8.4$ ), $7.62(\mathrm{~d}, 2 \mathrm{H}, J=8.4), 7.70(\mathrm{~s}, 1 \mathrm{H})$, 8.08-8.18 (m, 2H). ${ }^{13} \mathrm{C}$ NMR $\left(75 \mathrm{MHz}, \mathrm{CDCl}_{3}\right) \delta 19.9,115.2$, 115.8, 116.8, 117.1, 124.5, 129.7, 131.3, 136.1, 156.6, 159.4, 162.4, 165.7. Anal. found, C, 68.39; H, 4.07; N, 9.40. $\mathrm{C}_{17} \mathrm{H}_{12} \mathrm{ClFN}_{2}$ requires $\mathrm{C}, 68.35$; $\mathrm{H}, 4.05$; $\mathrm{N}, 9.38$.

3-(4-Chlorophenyl)-6-(4-methoxyphenyl)-4-methylpyridazine (27): white crystals; $78 \%$; $\mathrm{mp} 150{ }^{\circ} \mathrm{C}$. IR $(\mathrm{KBr}): v_{\max }=3070$, 3050, 3000, 2970, 2840, 1608, 1585, 1487, 1392, 1091, 1017, $824 \mathrm{~cm}^{-1} .{ }^{1} \mathrm{H}$ NMR $\left(300 \mathrm{MHz}, \mathrm{CDCl}_{3}\right) \delta 2.45(\mathrm{~s}, 3 \mathrm{H}), 3.92(\mathrm{~s}, 3 \mathrm{H})$, 7.05 (d, 2H, $J=8.4$ ), 7.49 (d, 2H, $J=8.4$ ), 7.62 (d, 2H, $J=8.4$ ), 7.68 (s, $1 \mathrm{H}), 8.11$ (d, $2 \mathrm{H}, J=8.1) \cdot{ }^{13} \mathrm{C}$ NMR (75 MHz, $\left.\mathrm{CDCl}_{3}\right) \delta$ 20.0, 56.0, 113.2, 113.8, 114.1, 115.5, 127.5, 129.2, 129.9, 131.3, 135.5, 135.9, 157.1, 158.9. Anal. found, C, 69.59; H, 4.88; N, 9.13. $\mathrm{C}_{18} \mathrm{H}_{15} \mathrm{ClN}_{2} \mathrm{O}$ requires $\mathrm{C}, 69.57 ; \mathrm{H}, 4.86 ; \mathrm{N}, 9.01$.

3-(4-Chlorophenyl)-6-(3-bromophenyl)-4-methylpyridazine (28): yellow crystals; $71 \%$; mp $132{ }^{\circ} \mathrm{C}$. IR (KBr): $v_{\max }=3091$, 3056, 3011, 2971, 2932, 1567, 1487, 1376, 1091, 1001, 843 $\mathrm{cm}^{-1} .{ }^{1} \mathrm{H} \mathrm{NMR}\left(300 \mathrm{MHz}, \mathrm{CDCl}_{3}\right) \delta 2.45(\mathrm{~s}, 3 \mathrm{H}), 7.40(\mathrm{t}, 1 \mathrm{H}, J=$ 7.8), $7.50(\mathrm{~d}, 2 \mathrm{H}, J=8.4$ ), 7.57-7.67 (m, 3H), $7.72(\mathrm{~s}, 1 \mathrm{H}), 8.07$ $(\mathrm{d}, 1 \mathrm{H}, J=7.8), 8.29(\mathrm{~s}, 1 \mathrm{H}) .{ }^{13} \mathrm{C} \mathrm{NMR}\left(75 \mathrm{MHz}, \mathrm{CDCl}_{3}\right) \delta 20.0$, $124.9,126.3,127.6,129.2,129.5,130.8,131.3,131.8,133.6$, 135.2, 136.3, 138.1, 156.2, 159.8. Anal. found, C, 56.80; H, 3.38;
$\mathrm{N}, 7.90 . \mathrm{C}_{17} \mathrm{H}_{12} \mathrm{BrClN}_{2}$ requires $\mathrm{C}, 56.77 ; \mathrm{H}, 3.36 ; \mathrm{N}, 7.79$.

3-(4-Chlorophenyl)-6-(3-methoxyphenyl)-4-methylpyridazine (29): white crystals, $77 \%, \mathrm{mp} 150{ }^{\circ} \mathrm{C}$. IR (KBr): $v_{\max }=3007$, 2936, 2835, 1595, 1581, 1493, 1390, 1255, 1174, 1035, 843, $795 \mathrm{~cm}^{-1} .{ }^{1} \mathrm{H}-\mathrm{NMR}\left(300 \mathrm{MHz}, \mathrm{CDCl}_{3}\right) \delta 2.45(\mathrm{~s}, 3 \mathrm{H}), 3.92(\mathrm{~s}, 3 \mathrm{H})$, $7.06(\mathrm{~d}, J=8.4 \mathrm{~Hz}, 1 \mathrm{H}), 7.44(\mathrm{t}, J=8.4 \mathrm{~Hz}, 1 \mathrm{H}), 7.51(\mathrm{~d}, J=8.7 \mathrm{~Hz}$, 2H), 7.64-7.61 (m, 3H), $7.74(\mathrm{~s}, 1 \mathrm{H}), 7.81(\mathrm{~s}, 1 \mathrm{H}) .{ }^{13} \mathrm{C}$ NMR $(75$ $\left.\mathrm{MHz}, \mathrm{CDCl}_{3}\right) \delta 19.8,54.6,110.9,120.2,124.7,127.5,128.9$, 129.5, 129.8, 131.0, 131.6, 135.4, 136.0, 137.5, 157.3, 159.5. Anal. found, $\mathrm{C}, 69.60 ; \mathrm{H}, 4.84 ; \mathrm{N}, 9.21$. $\mathrm{C}_{18} \mathrm{H}_{15} \mathrm{ClN}_{2} \mathrm{O}$ requires $\mathrm{C}$, 69.57; H, 4.86; N, 9.01 .

3-(4-Chlorophenyl)-6-(3,4-dimethoxyphenyl)-4-methylpyridazine (30): white crystals; $78 \%$; $\operatorname{mp} 182{ }^{\circ} \mathrm{C}$. IR (KBr): $v_{\max }=$ 3086, 3061, 3001, 2966, 2942, 2902, 2842, 2882, 1587, 1464, 1240, 1091, 1023, $838 \mathrm{~cm}^{-1}$. ${ }^{1} \mathrm{H}$ NMR (300 MHz, $\left.\mathrm{CDCl}_{3}\right) \delta 2.42$ (s, 3H), 3.95 (s, 3H), $4.00(\mathrm{~s}, 3 \mathrm{H}), 6.98(\mathrm{~d}, 1 \mathrm{H}, J=8.4), 7.47(\mathrm{~d}$, $2 \mathrm{H}, J=7.8), 7.51-7.64(\mathrm{~m}, 3 \mathrm{H}), 7.69(\mathrm{~s}, 1 \mathrm{H}), 7.93(\mathrm{~s}, 1 \mathrm{H}) .{ }^{13} \mathrm{C}$ NMR (75 MHz, $\left.\mathrm{CDCl}_{3}\right) \delta$ 20.0, 55.3, 56.6, 108.8, 109.1, 109.8, 110.7, 111.3, 111.7, 125.6, 127.7, 128.6, 131.3, 135.5, 135.9, 156.9, 158.9. Anal. found, C, 66.99; $\mathrm{H}, 5.04 ; \mathrm{N}, 8.31$. $\mathrm{C}_{17} \mathrm{H}_{17} \mathrm{ClN}_{2} \mathrm{O}_{2}$ requires $\mathrm{C}, 66.96 ; \mathrm{H}, 5.03 ; \mathrm{N}, 8.22$.

3-(4-Chlorophenyl)-6-(3,4-methylenedioxyphenyl)-4-methylpyridazine (31): yellow crystals, $80 \%, \mathrm{mp} 166^{\circ} \mathrm{C}$. IR (KBr): $v_{\max }$ $=3081,3046,3006,2966,2897,2787,1592,1505,1452,1108$, 1001, $819 \mathrm{~cm}^{-1}$. ${ }^{1} \mathrm{H}$ NMR (300 MHz, $\left.\mathrm{CDCl}_{3}\right) \delta 2.42(\mathrm{~s}, 3 \mathrm{H}), 6.05$ $(\mathrm{s}, 2 \mathrm{H}), 6.94(\mathrm{~d}, J=8.1,1 \mathrm{H}), 7.48(\mathrm{~d}, J=8.1,2 \mathrm{H}), 7.66-7.54(\mathrm{~m}$, $4 \mathrm{H}), 7.71(\mathrm{~s}, 1 \mathrm{H}) .{ }^{13} \mathrm{C}$ NMR $\left(75 \mathrm{MHz}, \mathrm{CDCl}_{3}\right) \delta 19.9,101.5,106.6$, 107.4, 108.8, 109.3, 125.7, 127.5, 129.7, 130.3, 131.3, 135.5, 135.9, 148.5, 157.0, 159.0. Anal. found, C, 66.59; H, 4.04; N, 8.81. $\mathrm{C}_{18} \mathrm{H}_{13} \mathrm{ClN}_{2} \mathrm{O}_{2}$ requires $\mathrm{C}, 66.57 ; \mathrm{H}, 4.03 ; \mathrm{N}, 8.63$.

3-(4-Chlorophenyl)-6-(4-hydroxy-3-methoxyphenyl)-4methylpyridazine (32): white crystals; 78\%; mp $150{ }^{\circ} \mathrm{C}$. IR $(\mathrm{KBr}): v_{\max }=3529,3078,3049,2939,1596,1510,1489,1270$, 1029, 824, $789 \mathrm{~cm}^{-1} .{ }^{1} \mathrm{H}$ NMR (300 MHz, $\left.\mathrm{CDCl}_{3}\right) \delta 2.44(\mathrm{~s}, 3 \mathrm{H})$, $4.01(\mathrm{~s}, 3 \mathrm{H}), 6.01(\mathrm{~s}, 1 \mathrm{H}), 7.05(\mathrm{~d}, 1 \mathrm{H}, J=8.1), 7.51-7.47(\mathrm{~m}, 3 \mathrm{H})$, 7.62 (d, 2H, $J=8.1), 7.70(\mathrm{~s}, 1 \mathrm{H}), 7.99(\mathrm{~s}, 1 \mathrm{H}) .{ }^{13} \mathrm{C}$ NMR $(75 \mathrm{MHz}$, $\left.\mathrm{CDCl}_{3}\right) \delta 55.2,56.9,108.5,110.4,113.5,115.6,118.9,120.8$, $121.1,123.9,125.7,127.4,129.4,129.7,131.5,135.9$. Anal. found, $\mathrm{C}, 66.17 ; \mathrm{H}, 4.61 ; \mathrm{N}, 8.76 . \mathrm{C}_{18} \mathrm{H}_{15} \mathrm{ClN}_{2} \mathrm{O}_{2}$ requires $\mathrm{C}, 66.16$; H, 4.63; N, 8.57.

3-(4-Methylphenyl)-6-phenyl-4-methylpyridazine (33): white crystals; $70 \%$; $\operatorname{mp~} 133{ }^{\circ} \mathrm{C}$. IR $(\mathrm{KBr}): v_{\max }=3055,2930,1586$, 1490, 1392, 1092, 10018, $839 \mathrm{~cm}^{-1} .{ }^{1} \mathrm{H}$ NMR (300 MHz, $\mathrm{CDCl}_{3}$ ) $\delta 2.45(\mathrm{~s}, 3 \mathrm{H}), 2.46(\mathrm{~s}, 3 \mathrm{H}), 7.33(\mathrm{~d}, 2 \mathrm{H}, J=7.8), 7.45-7.60(\mathrm{~m}$, $5 \mathrm{H}), 7.74(\mathrm{~s}, 1 \mathrm{H}), 8.13(\mathrm{~d}, 2 \mathrm{H}, J=6.3) .{ }^{13} \mathrm{C} \mathrm{NMR}\left(75 \mathrm{MHz}, \mathrm{CDCl}_{3}\right)$ $\delta$ 20.0, 20.1, 125.0, 126.2, 126.6, 127.9, 128.1, 129.6, 130.0, 130.5, 136.5, 138.9, 157.2, 160.4. Anal. found, C, 83.07; H, 6.20; $\mathrm{N}, 10.91 . \mathrm{C}_{18} \mathrm{H}_{16} \mathrm{~N}_{2}$ requires $\mathrm{C}, 83.04 ; \mathrm{H}, 6.19 ; \mathrm{N}, 10.76$.

3-(4-Methylphenyl)-6-(4-bromophenyl)-4-methylpyridazine (34): white crystals; 94\%; mp $166^{\circ} \mathrm{C}$. IR (KBr): $v_{\max }=3090$, 2922, 2850, 1588, 1486, 1407, 1073, 1003, $821 \mathrm{~cm}^{-1}$. ${ }^{1} \mathrm{H}$ NMR $\left(300 \mathrm{MHz}^{\mathrm{CDCl}}\right) \delta 2.45(\mathrm{~s}, 3 \mathrm{H}), 2.48(\mathrm{~s}, 3 \mathrm{H}), 7.33(\mathrm{~d}, 2 \mathrm{H}, J=$ 7.5), 7.57 (d, 2H, $J=7.5$ ), $7.66(\mathrm{~d}, 2 \mathrm{H}, J=8.1$ ), $7.75(\mathrm{~s}, 1 \mathrm{H}), 8.03$ $(\mathrm{d}, 2 \mathrm{H}, J=8.1) .{ }^{13} \mathrm{C} \mathrm{NMR}\left(75 \mathrm{MHz}, \mathrm{CDCl}_{3}\right) \delta 20.1,21.3,124.8$, 127.7, 128.2, 128.5, 129.5, 129.9, 130.1, 130.4, 131.4, 133.0, 156.2, 160.5. Anal. found, C, 63.78; $\mathrm{H}, 4.45 ; \mathrm{N}, 8.30 . \mathrm{C}_{18} \mathrm{H}_{15} \mathrm{BrN}_{2}$ requires $\mathrm{C}, 63.73 ; \mathrm{H}, 4.46 ; \mathrm{N}, 8.26$. 
3-(4-Methylphenyl)-6-(4-chlorophenyl)-4-methylpyridazine (35): yellow crystals; $68 \%$; $\mathrm{mp} 184{ }^{\circ} \mathrm{C}$. IR (KBr): $v_{\max }=3090$, $3035,1918,1586,1438,1406,1090,1008,824 \mathrm{~cm}^{-1}$. ${ }^{1} \mathrm{H} \mathrm{NMR}$ $\left(300 \mathrm{MHz}, \mathrm{CDCl}_{3}\right) \delta 2.45(\mathrm{~s}, 3 \mathrm{H}), 2.47(\mathrm{~s}, 3 \mathrm{H}), 7.33(\mathrm{~d}, 2 \mathrm{H}, J=$ 7.8), 7.45-7.6 (m, 4H), 7.73 (s, 1H), 8.08 (d, 2H, J = 8.7). ${ }^{13} \mathrm{C}$ NMR $\left(75 \mathrm{MHz} \mathrm{CDCl}_{3}\right) \delta 20.1,21.3,125.0,126.6,127.4,128.2,129.2$, $129.8,130.0,130.3,136.9,139.2,156.1,160.5$. Anal. found, C, 73.39; $\mathrm{H}, 5.18 ; \mathrm{N}, 9.66 . \mathrm{C}_{18} \mathrm{H}_{15} \mathrm{ClN}_{2}$ requires $\mathrm{C}, 73.34 ; \mathrm{H}, 5.13 ; \mathrm{N}$, 9.50 .

3-(4-Methylphenyl)-6-(4-fluorophenyl)-4-methylpyridazine (36): white crystals; 69\%; mp $175^{\circ} \mathrm{C}$. IR (KBr): $v_{\max }=3075$, 2920, 1590, 1506, 1378, 1222, 1099, $818 \mathrm{~cm}^{-1}$. ${ }^{1} \mathrm{H}$ NMR $(300$ $\left.\mathrm{MHz} \mathrm{CDCl}_{3}\right) \delta 2.45(\mathrm{~s}, 3 \mathrm{H}), 2.47(\mathrm{~s}, 3 \mathrm{H}), 7.17-7.29(\mathrm{~m}, 2 \mathrm{H}), 7.33$ (d, 2H, $J=7.8$ ), 7.56 (d, 2H, $J=7.8), 7.70(\mathrm{~s}, 1 \mathrm{H}), 8.09-8.18(\mathrm{~m}$, 2H). ${ }^{13} \mathrm{C}$ NMR $\left(75 \mathrm{MHz}, \mathrm{CDCl}_{3}\right) \delta 20.1,21.3,115.2,116.1,116.7$, 117.0, 126.1, 128.1, 128.3, 129.9, 156.3, 160.4, 162.4, 165.7 Anal. found, C, 77.70; $\mathrm{H}, 5.46 ; \mathrm{N}, 10.20 . \mathrm{C}_{18} \mathrm{H}_{15} \mathrm{FN}_{2}$ requires $\mathrm{C}$, 77.68; $\mathrm{H}, 5.43 ; \mathrm{N}, 10.07$.

3-(4-Methylphenyl)-6-(4-methoxyphenyl)-4-methylpyridazine (37): yellow crystals; $75 \%$; mp $134{ }^{\circ} \mathrm{C}$. IR (KBr): $v_{\max }=3005$, 2925, 2835, 1609, 1583, 1395, 1256, 1023, $845 \mathrm{~cm}^{-1}$. ${ }^{1} \mathrm{H}$ NMR $\left(300 \mathrm{MHz}, \mathrm{CDCl}_{3}\right) \delta 2.40(\mathrm{~s}, 3 \mathrm{H}), 2.44(\mathrm{~s}, 3 \mathrm{H}), 3.88(\mathrm{~s}, 3 \mathrm{H}), 7.04$ (d, 2H, $J=8.1$ ), 7.31 (d, 2H, $J=7.8$ ), 7.56 (d, 2H, $J=7.8$ ), 7.69 (s, $1 \mathrm{H}), 8.11(\mathrm{~d}, 2 \mathrm{H}, J=8.7) .{ }^{13} \mathrm{C} \mathrm{NMR}\left(7.5 \mathrm{MHz}, \mathrm{CDCl}_{3}\right) \delta 20.1,21.3$, 56.0, 113.3, 113.7, 115.0, 115.5, 126.0, 127.6, 128.1, 130.0, 138.9, 156.7, 159.8, 161.2. Anal. found, C, 78.57; H, 6.24; N, 9.77. $\mathrm{C}_{19} \mathrm{H}_{18} \mathrm{~N}_{2} \mathrm{O}$ requires $\mathrm{C}, 78.59 ; \mathrm{H}, 6.25 ; \mathrm{N}, 9.65$.

3-(4-Methylphenyl)-6-(3,4-dimethoxyphenyl)-4-methylpyridazine (38): white crystals; $61 \%$; mp $170{ }^{\circ} \mathrm{C}$. IR (KBr): $v_{\max }=$ $3005,2940,2840,1605,1588,1422,1259,1025,821 \mathrm{~cm}^{-1}{ }^{1}{ }^{\mathrm{H}}$ NMR (300 MHz, $\left.\mathrm{CDCl}_{3}\right) \delta 2.44(\mathrm{~s}, 3 \mathrm{H}), 2.46(\mathrm{~s}, 3 \mathrm{H}), 3.94(\mathrm{~s}, 3 \mathrm{H})$, $4.01(\mathrm{~s}, 3 \mathrm{H}), 6.98(\mathrm{~d}, 1 \mathrm{H}, J=8.4), 7.31$ (d, 2H, $J=7.5), 7.48-7.64$ $(\mathrm{m}, 3 \mathrm{H}), 7.74(\mathrm{~s}, 1 \mathrm{H}), 7.96(\mathrm{~s}, 1 \mathrm{H}) .{ }^{13} \mathrm{C} \mathrm{NMR}\left(75 \mathrm{MHz}, \mathrm{CDCl}_{3}\right) \delta$ 20.2, 21.3, 55.2, 56.7, 109.2, 109.9, 110.4, 110.9, 11.8, 112.2, $126.4,128.5,129.9,139.0,149.5,151.0,156.4,159.9$. Anal. found, $\mathrm{C}, 75.00 ; \mathrm{H}, 6.32 ; \mathrm{N}, 8.84 . \mathrm{C}_{20} \mathrm{H}_{20} \mathrm{~N}_{2} \mathrm{O}_{2}$ requires $\mathrm{C}, 74.98$; H, 6.29; N, 8.74.

\section{Results and discussion}

During our research program on the synthesis of aryl-substituted pyridazine derivatives [25], we used the reaction of 3-bromopropiophenone (39a) with phenylglyoxalmonohydrate (40a) and hydrazine hydrate in water as a model reaction. This one-pot system afforded hydrazone compounds as by-products at room temperature, $50{ }^{\circ} \mathrm{C}$ and at reflux (Table 1 , entries 1-3). This could be a reflection of the low acidity of the $\alpha$-hydrogens on propiophenone, and so we investigated the use of organic bases such as DABCO, DBU and DBN to catalyze this reaction. With an initial concentration of $10 \mathrm{~mol} \%$ of either DABCO, DBU or DBN at $25{ }^{\circ} \mathrm{C}$ for $6 \mathrm{~h}$, the desired pyridazine derivative was afforded in 25\%,15\% and $10 \%$ yield, respectively (Table 1, entries 4-6). The influence of solvent was also investigated, and the results are summarized in Table 1 . No product was isolated when the reaction was carried out in pure EtOH (entries 7-9), and a much longer time was needed when
Table 1

Optimization of the reaction conditions.

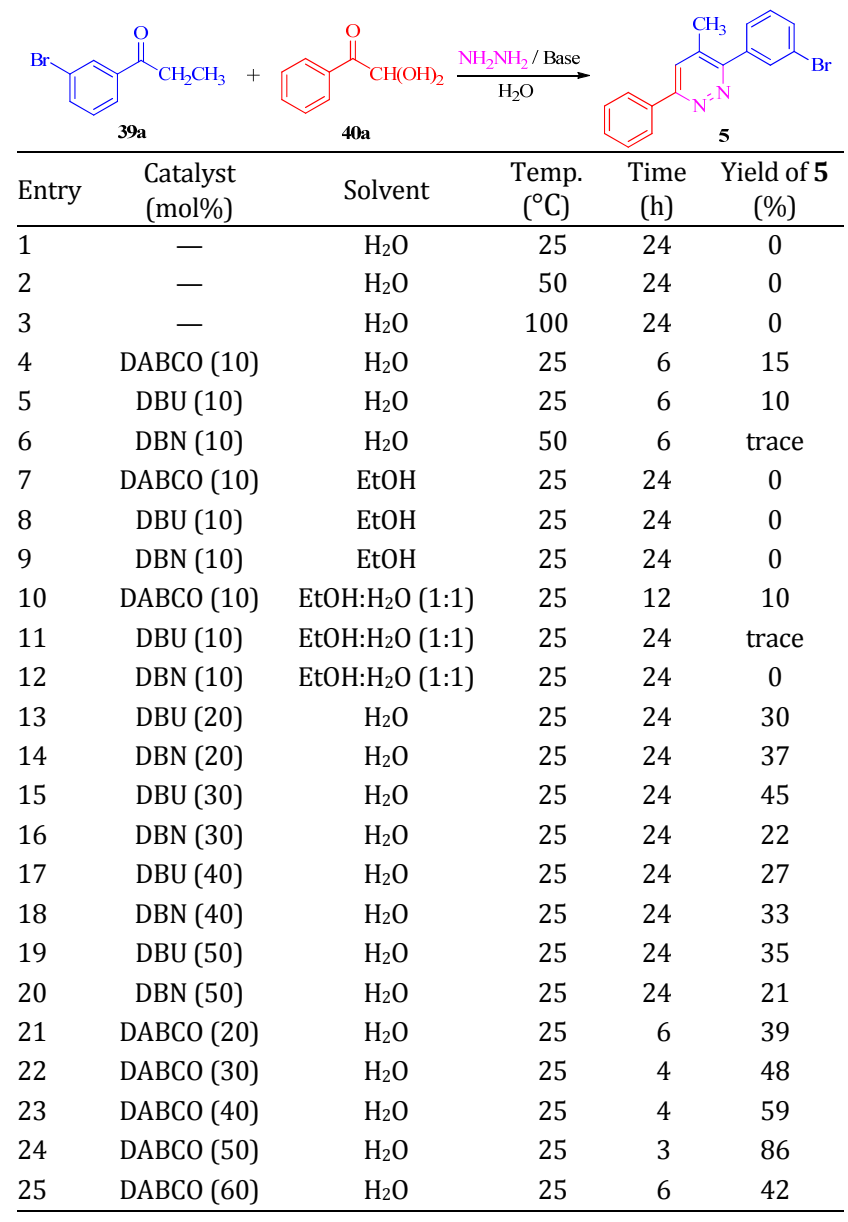

the reaction was carried out in a 1:1 ratio of $\mathrm{EtOH}: \mathrm{H}_{2} \mathrm{O}$ (entries 10-12). Attempts to improve the reaction conditions by running the reaction in $\mathrm{H}_{2} \mathrm{O}$, EtOH and $\mathrm{H}_{2} \mathrm{O}$ : EtOH (1:1) in the presence of $10 \mathrm{~mol} \%$ of the organic bases at $50{ }^{\circ} \mathrm{C}$ failed. Monitoring the reaction progress via TLC showed that an increase in the reaction temperature led to an increase in by-product formation, and no pyridazine products were formed. The above results indicated that $\mathrm{H}_{2} \mathrm{O}$ accelerated the reaction, and it was found to the best solvent for this reaction at room temperature $\left(25^{\circ} \mathrm{C}\right)$. Encouraged by these results, we continued to focus on optimizing the amounts of the basic organocatalysts for the reaction. Tuning the catalyst concentration revealed that increasing the amounts of DBU and DBN from 20 to $40 \mathrm{~mol} \%$ led to a small increase in the yield (Table 1, entries 13-18). Using $50 \mathrm{~mol} \%$ of both DBU and DBN caused a decrease in yield (Table 1, entries 19 and 20). Surprisingly, in the case of DABCO, an increase in concentration caused a significant increase in the yield of the pyridazine product (Table 1, entries 21-23), and adding $50 \mathrm{~mol} \%$ of DABCO to the reaction mixture led to the best result (Table 1, entry 24). Further increases in the molar ratio of DABCO did not improve the reaction yield and, in contrast, it caused a significant decrease in the efficiency of the reaction (Table 1 , entry 25 ). 
With the optimized reaction conditions in hand, we moved on to examine the substrate scope using a wide variety of propiophenones and arylglyoxal monohydrates (Table 2). Arylgly- oxalmonohydrates bearing electron-withdrawing groups gave better yields, in shorter reaction times, than the arylglyoxalmonohydrates bearing electron-donating groups. It is worth-

Table 2

Substrate scope study using different propiophenones and arylglyoxalmonohydrates.

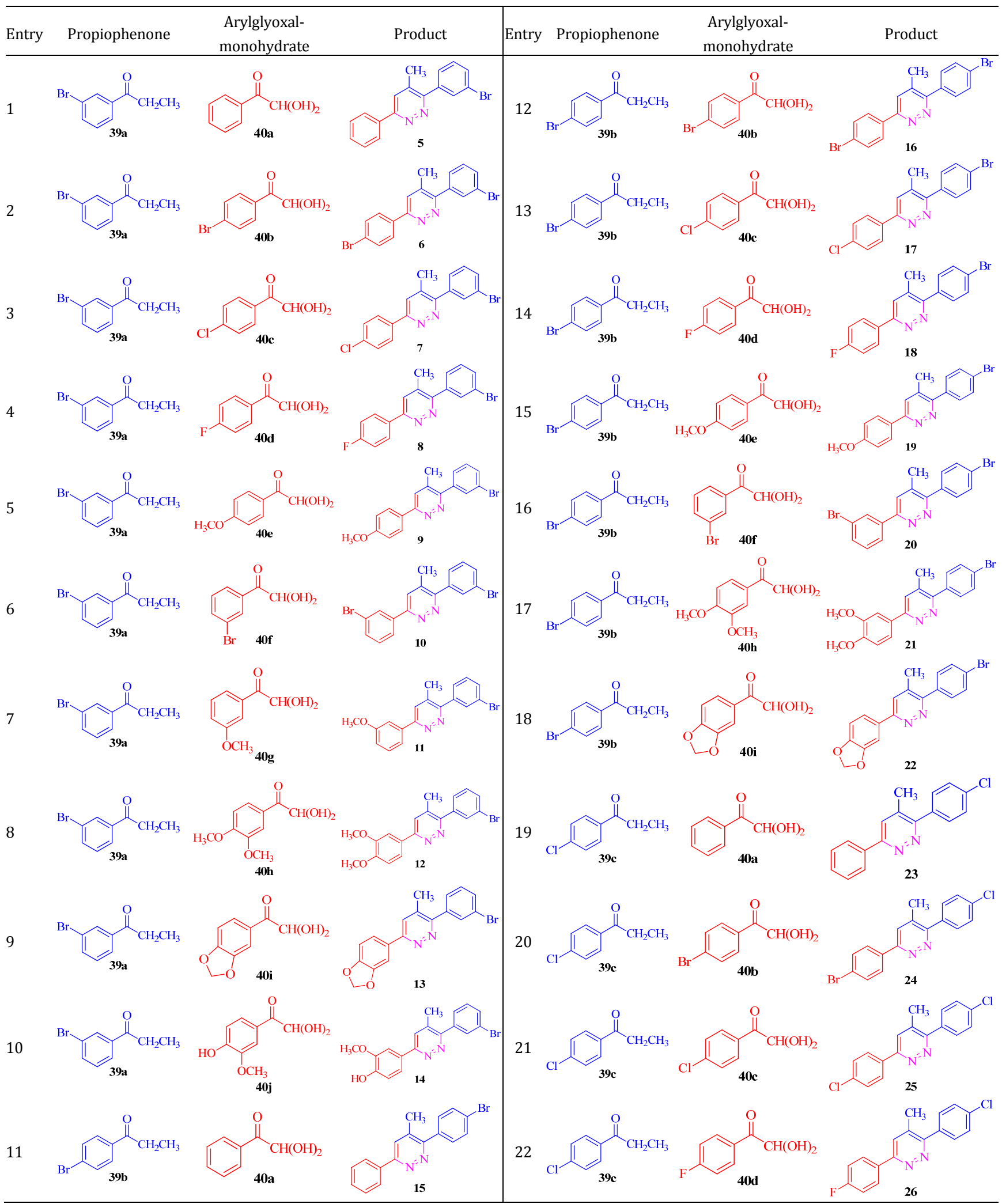


Table 2 (continued)

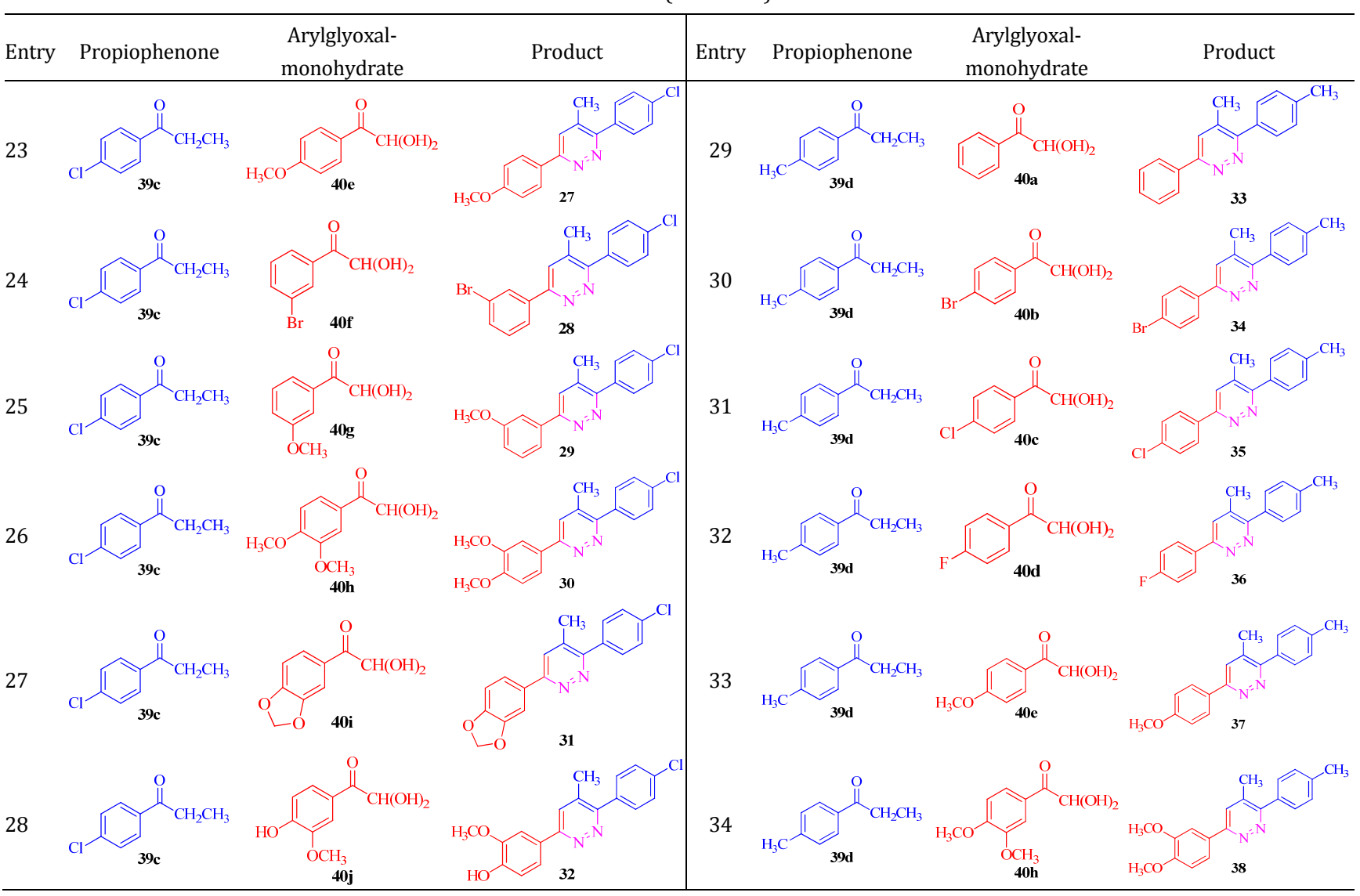

while to note that all of the reactions proceeded selectively to generate the 3,6-diaryl-4-methylpyridazine as a single regioisomer.

Full characterization including IR, ${ }^{1} \mathrm{H}$ NMR, ${ }^{13} \mathrm{C}$ NMR and elemental analysis proved the identity of all the pyridazine products 5-38. The diagnostic singlet around $\delta \approx 7.70$ in the ${ }^{1} \mathrm{H}$ NMR spectra was ascribed to the $\mathrm{C}_{5}-\mathrm{H}$ of the pyridazine ring. All of the compounds 5-38 are believed to be the only regioisomers present, and no evidence for the formation of the other isomer was observed for all new pyridazine derivatives. As

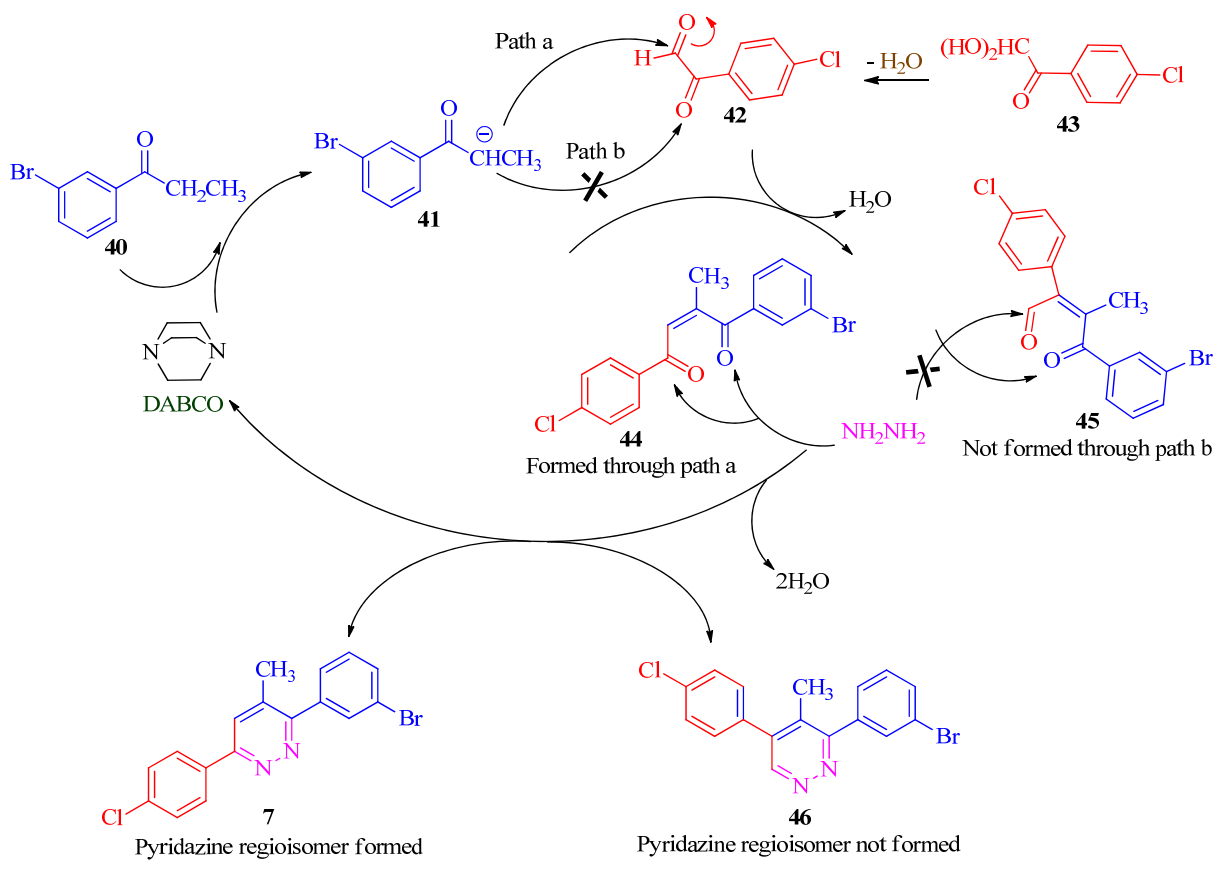

Scheme 2. Proposed mechanism for the regioselective DABCO-catalyzed synthesis of 3,6-diaryl-4-methylpyridazine derivatives. 
the formyl group is more electrophilic than the keto group in the arylglyoxal scaffold [25-30] (Scheme 2), the regioselectivity is due to carbanion $\mathbf{4 1}$ attacking the formyl group of arylglyoxal 42, leading to the formation of Knoevenagel adduct 44 through path a. In the IR spectra, the characteristic absorption band at $1580 \mathrm{~cm}^{-1}$ can be assigned to the $\mathrm{C}=\mathrm{N}$ bonds of the pyridazine ring. In the ${ }^{13} \mathrm{C} \mathrm{NMR}$, two signals located at the lowest fields, between $\delta \approx 156-159$, were assigned as the carbon atoms of the corresponding $\mathrm{C}=\mathrm{N}$ groups. Efforts toward preparing a single crystal from these substituted pyridazines is ongoing.

\section{Conclusions}

We have developed an eco-friendly, regioselective and highly efficient three component base-organocatalyzed reaction involving various arylglyoxalmonohydrates, substituted propiophenones and hydrazine hydrate, to produce novel 3,6-diaryl-4-methylpyridazines bearing diverse aryl substituents. Mild reaction conditions, operational simplicity and facile workup are the main advantages of this synthetic strategy.

\section{Acknowledgments}

This work was partially supported by the Research Council of Payame Noor University.

\section{References}

[1] G. P. Ellis, in: Synthesis of Fused Heterocycles, John Wiley and Sons Inc., New York, 1987, 226-239.

[2] T. J. Ritchie, S. J. F. Macdonald, S. Peace, S. D. Pickett, C. N. Luscombe, MedChemComm, 2012, 3, 1062-1069.

[3] C. G. Wermuth, MedChemComm, 2011, 2, 935-941.

[4] D. L. Boger, Chem. Rev., 1986, 86, 781-794.

[5] D. L. Boger, M. Patel, Prog. Heterocycl. Chem., 1989, 1, 30-64.

[6] J. Sauer, in: A. R. Katritzky, C. W. Rees, E. F. V. Scriven eds., Comprehensive Heterocyclic Chemistry II: A Review of the Literature 1982-1995, Pergamon, London, 1996, Vol. 6, 901-955.
[7] J. J. Bourguignon, S. Oumouch, M. Schmitt, Curr. Org. Chem., 2006, $10,277-295$.

[8] C. G. Wermuth, G. Schlewer, J. J. Bourguignon, G. Maghioros, M. J. Bouchet, C. Moire, J. P. Kan, P. Worms, K. Biziere, J. Med. Chem., 1989, 32, 528-537.

[9] A. Volonterio, L. Moisan, J. Rebek Jr., Org. Lett., 2007, 9, 3733-3736.

[10] E. Isabel, D. A. Powell, W. C. Black, C. C. Chan, S. Crane, R. Gordon, J. Guay, S. Guiral, Z. Huang, J. Robichaud, K. Skorey, P. Tawa, L. J. Xu, L. Zhang, R. Oballa, Bioorg. Med. Chem. Lett., 2011, 21, 479-483.

[11] Z. H. Wan, A. Hall, Y. Jin, J. N. Xiang, E. Yang, A. Eatherton, B. Smith, G. Yang, H. H. Yu, J. Wang, L. Ye, L. F. Lau, T. Yang, W. Mitchell, W. Cai, X. M. Zhang, Y. X. Sang, Y. H. Wang, Z. L. Tong, Z. Q. Cheng, I. Hussain, J. D. Elliott, Y. Matsuoka, Bioorg. Med. Chem. Lett., 2011, 21, 4016-4019.

[12] R. J. Gleave, P. J. Beswick, A. J. Brown, G. M. P. Giblin, P. Goldsmith, C. P. Haslam, W. L. Mitchell, N. H. Nicholson, L. W. Page, S. Patel, S. Roomans, B. P. Slingsby, M. E. Swarbrick, Bioorg. Med. Chem. Lett., 2010, 20, 465-468.

[13] T. Asano, H. Yamazaki, C. Kasahara, H. Kubota, T. Kontani, Y. Harayama, K. Ohno, H. Mizuhara, M. Yokomoto, K. Misumi, T. Kinoshita, M. Ohta, M. Takeuchi, J. Med. Chem., 2012, 55, 7772-7785.

[14] J. Sapi, J. Y. Laronze, Arkivoc, 2004, (vii), 208-222.

[15] J. P. Zhu, H. Bienayme, Multicomponent Reactions, Wiley-VCH, Weinheim, 2005.

[16] N. Hazeri, M. T. Maghsoodlou, S. M. Habibi-Khorassani, M. Ziyaadini, G. Marandi, K. Khandan-Barani, H. R. Bijanzadeh, Arkivoc, 2007, (xiii), 34-40.

[17] A. Dömling, B. Beck, E. Herdtweck, W. Antuch, C. Oefner, N. Yehia, A. Gracia-Marques, Arkivoc, 2007, (xii), 99-109.

[18] D. J. Ramon, M. Yus, Angew. Chem. Int. Ed., 2005, 44, 1602-1634.

[19] A. Basso, L. Banfi, R. Riva, G. Guanti, J. Org. Chem., 2005, 70, 575-579.

[20] L. Chen, Y. Du, X. P. Zeng, T. D. Shi, F. Zhou, J. Zhou, Org. Lett., 2015, $17,1557-1560$.

[21] L. Chen, T. D. Shi, J. Zhou, Chem. Asian J., 2013, 8, 556-559.

[22] M. Tisler, R. Stanovnik, Adv. Heterocycl. Chem., 1968, 9, 211-320.

[23] M. D. Bezoari, W. W. Paudler, J. Org. Chem., 1980, 45, 4584-4586.

[24] C. G. Wermuth, G. Schlewer, J. J. Bourguignon, G. Maghioros, M. J. Bouchet, C. Moire, J. P. Kan, P. Worms, K. Biziere, J. Med. Chem., 1989, 32, 528-537.

\section{Graphical Abstract}

Chin. J. Catal., 2016, 37: 517-525 doi: 10.1016/S1872-2067(15)61060-9

An environmentally-friendly base organocatalyzed one-pot strategy for the regioselective synthesis of novel 3,6-diaryl-4-methylpyridazines

Mehdi Rimaz*, Farkhondeh Aali

Payame Noor University, Iran
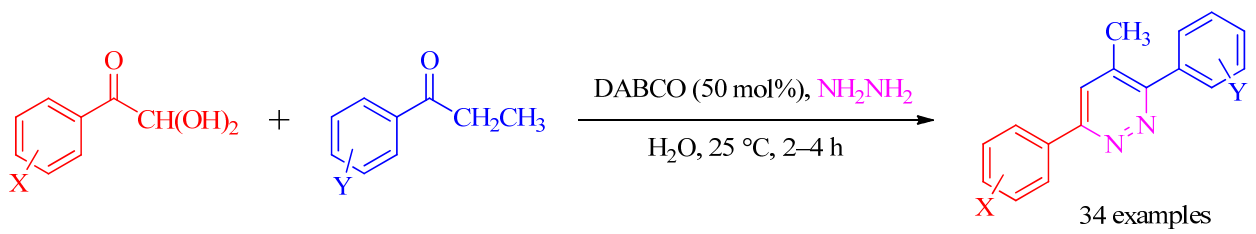

$\mathrm{X}=4-\mathrm{H}, 4-\mathrm{Br}, 4-\mathrm{Cl}, 4-\mathrm{F}, 4-\mathrm{OCH}_{3}, 3-\mathrm{Br}, 3-\mathrm{OCH}_{3}, 3,4-\left(\mathrm{OCH}_{3}\right)_{2}, 3,4-\left(\mathrm{OCH}_{2} \mathrm{O}\right), 4-\mathrm{OH}-3-\mathrm{OCH}_{3}$ $\mathrm{Y}=4-\mathrm{Br}, 3-\mathrm{Br}, 4-\mathrm{Cl}, 4-\mathrm{CH}_{3}$

This work describes the regioselective synthesis of novel 3,6-diaryl-4-methylpyridazines directly from a DABCO-catalyzed three-component condensation of propiophenones, arylglyoxalmonohydrates and hydrazine hydrate in water. 
[25] M. Rimaz, J. Khalafy, Arkivoc, 2010, (ii), 110-117.

[26] M. Rimaz, Z. Jalalian, H. Mousavi, R. H. Prager, Tetrahedron Lett., 2016, 57, 105-109.

[27] M. Rimaz, Aust. J. Chem., 2015, 68, 1529-1534.

[28] M. Rimaz, P. Pourhossein, B. Khalili, Turk. J. Chem., 2015, 39,
$244-254$.

[29] M. Rimaz, H. Rabiei, B. Khalili, R. H. Prager, Aust. J. Chem., 2014, 67, 283-288.

[30] M. Rimaz, A. Mishokraie, B. Khalili, P. Motiee, Arkivoc, 2015, (v), 88-98.

\section{环境友好碱有机催化一锅法区域选择性合成新型3,6-二芳基-4-甲基哒嗪 \\ Mehdi Rimaz , Farkhondeh Aali \\ 帕亚莫·努尔大学化学系, 德黑兰19395-3697, 伊朗}

摘要: 报道了一种新型三组分反应策略用于区域选择性合成一系列三取代的哒嗪, 即于水中进行DABCO催化的苯丙酮、 芳香乙二醛一水合物和水合肼三组分缩合反应. 该法提供了一种绿色便利的一锅法制备各种芳基取代的3,6-二芳基-4-甲 基哒嗪, 它以水为溶剂, DABCO为绿色碱有机催化剂, 具有高区域选择性、操作简便、产物收率高和后处理简单等优点. 关键词: 1,4-二氮杂二环[2.2.2]辛烷; 苯丙酮; 芳香乙二醛一水合物; 哒嗪

收稿日期: 2016-01-07. 接受日期: 2016-01-29. 出版日期: 2016-04-05.

*通讯联系人. 电话: +98-443-5625711; 传真: +98-443-5666999; 电子信箱: rimaz.mehdi@gmail.com

本文的英文电子版由Elsevier出版社在ScienceDirect上出版(http://www.sciencedirect.com/science/journal/18722067). 